\title{
The effect of word predictability on the eye movements of Chinese readers
}

\author{
KEITH RAYNER, XINGSHAN LI, and BARBARA J. JUHASZ \\ University of Massachusetts, Amherst, Massachusetts \\ and \\ GUOLI YAN \\ Tianjin Normal University, Tianjin, China
}

\begin{abstract}
Eye movements of Chinese readers were monitored as they read sentences containing target words whose predictability from the preceding context was high, medium, or low. Readers fixated for less time on high- and medium-predictable target words than on low-predictable target words. They were also more likely to fixate on low-predictable target words than on high- or medium-predictable target words. The results were highly similar to those of a study by Rayner and Well (1996) with English readers and demonstrate that Chinese readers, like readers of English, exploit target word predictability during reading.
\end{abstract}

Although a large proportion of the world's population consists of readers of Chinese, much less is known about eye movements that occur during reading of this logographic script than about those that occur during reading of alphabetic writing systems (particularly English). Text written in Chinese is formed by strings of equally spaced boxlike symbols called characters. Historically, Chinese text was printed from top to bottom (with the columns printed from right to left). However, like English, Chinese is now most typically printed horizontally from left to right. Unlike English (and other alphabetic writing systems), Chinese is written without spaces between successive characters and words. Furthermore, individual characters differ greatly in terms of complexity because they vary in (1) the number of strokes per character, (2) the number of radicals (or certain combinations of strokes that denote semantic or phonological information), and (3) the manner of construction (i.e., radicals can be combined in different ways to form compound words). Basically, there are many visual details packed into a constant, box-shaped area for each character.

Characters differ in complexity, as well as in the frequency with which they are seen. And although the concept of a word is not so clearly defined in Chinese as it is in English (so that Chinese readers will disagree somewhat concerning where word boundaries are located), it is the case that Chinese words also differ in frequency. The Chinese characters are more like morphemes, and

This research was supported by Grant HD26765 from NIH and a Grant from Microsoft Corporation. We thank David Balota, Max Coltheart, Reinhold Kliegl, and an anonymous reviewer for helpful comments on an earlier draft. Correspondence concerning this article should be addressed to K. Rayner, Department of Psychology, University of Massachusetts, Amherst, MA 01003 (e-mail: rayner@psych.umass.edu). most words are made up of two characters, although some words consist of only one character and some consist of three or more characters. Finally, Chinese words, like English words, presumably vary in terms of how predictable they are from the preceding context. To what extent do character complexity, character frequency, word frequency, and word predictability influence eye movements in the reading of Chinese? This question is interesting in the context of recent models of eye movement control, such as E-Z Reader (Reichle, Pollatsek, Fisher, \& Rayner, 1998; Reichle, Rayner, \& Pollatsek, 2003) and SWIFT (Engbert, Longtin, \& Kliegl, 2002; Kliegl \& Engbert, 2003), which do a good job of simulating the eye movement behavior of readers of alphabetic writing systems. These models typically take as input information about the frequency and predictability of the words in a text to simulate eye movement behavior in reading. In the case of Chinese, character complexity, character frequency, word frequency, and word predictability could all be important factors influencing eye movements. Thus, it is important to understand the eye movement characteristics of Chinese readers, in order not only to understand Chinese reading per se, but also to determine the extent to which the models that have been developed might be able to account for the eye movements of Chinese readers. ${ }^{1}$

What is known about the eye movements of Chinese readers? First, average fixation durations tend to be very similar (about 225-250 msec) for readers of Chinese and English (Chen, Song, Lau, Wong, \& Tang, 2003; Rayner, 1998; Sun \& Feng, 1999). Second, not surprisingly, average saccades are much shorter in Chinese (about 2.6 characters) than in English (about 7-8 letters), since the information is more densely packed in Chinese (Chen et al., 2003). Third, regression rate appears to be slightly higher in Chinese (about 15\%) than in English (about 10\%) skilled readers (Chen et al., 2003; Rayner, 1998). 
Fourth, the probability of skipping a word tends to be higher in Chinese than in English (42\% vs. 20\%), according to Chen et al., although a recent study by Tsai, Lee, Tzeng, Hung, and Yen (2004) reported skipping rates of around $10 \%$ for Chinese readers. Finally, the perceptual span of Chinese readers extends 1 character to the left of fixation to 2-3 characters to the right when they are reading from left to right (Inhoff \& Liu, 1997, 1998; see also Chen \& Tang, 1998); ${ }^{2}$ in contrast, for English, the span extends 3-4 letters to the left of fixation to about 14-15 letters to the right of fixation (Rayner, 1998).

With respect to the four different character/word variables that could influence reading behavior, there is only scant data on Chinese. For English, it is well known that word frequency and word predictability have strong influences on the fixation time on a word (Rayner, 1998), and word predictability influences word skipping (Brysbaert \& Vitu, 1998; Rayner, 1998). ${ }^{3}$ For Chinese, it has been demonstrated that character complexity and word frequency influence fixation time on a word and also skipping (Yang \& McConkie, 1999). Chen et al. (2003) noted that regression analyses that they carried out on Chinese adults' eye movement data indicated that character complexity and frequency were more important than word frequency. They also reported regression analyses with children (second, fourth, and sixth graders) that showed basically the same thing. Clearly, more work is needed to examine more precisely the extent to which character complexity, character frequency, and word frequency influence the eye movements of Chinese readers.

In the present study, we focus on the effect of word predictability on Chinese readers' eye movements. Surprisingly, there appears to be no prior work on this variable, which, as was noted above, has been shown to have a robust effect on the eye movements of readers of English (Ehrlich \& Rayner, 1981; Rayner \& Well, 1996). The study we report here is quite similar to that of Rayner and Well. We constructed sentences that contained target words that varied in terms of their having high, medium, or low predictability from the prior context. Readers' eye movements were then recorded as they read these sentences. Would the same pattern that was obtained in English emerge in Chinese, or would the differences in writing systems render the effect of predictability more potent or less potent in Chinese?

Theoretically, it seems a priori possible that the reading process could be different for readers of Chinese than for readers of alphabetic writing systems such as English. On the one hand, the lack of spacing between words (and the fact that Chinese readers often disagree on where word boundaries are) might lead them to rely more heavily on contextual information than do readers of English. In this case, we would expect contextual constraint/predictability to have stronger effects in Chinese than is the case in English. On the other hand, because more information (relatively speaking) falls within the foveal region in Chinese than in English, Chinese readers might not exploit word predictability to the extent that readers of English do. In this case, we would expect contextual constraint/predictability to have weaker effects for Chinese readers than for English readers. Finally, if reading processes are similar for Chinese and English readers, we would expect that predictability would have similar effects across languages.

\section{METHOD}

\section{Participants}

Sixteen native Chinese speakers who were students at the University of Massachusetts were paid to participate in the experiment. All of them had normal or corrected-to-normal vision, and all were naive regarding the purpose of the experiment.

\section{Apparatus}

Eye movements were recorded by an SR EyeLink II eyetracker, which has a resolution of approximately $30^{\prime}$ of arc. The participants read the target sentences (which were printed horizontally from left to right) on a 19 -in. NEC Trinitron monitor connected to a $166-\mathrm{MHz}$ Pentium PC. The participants wore a lightweight helmet that is part of the eye-tracking system. The eye-tracking system samples at the rate of $500 \mathrm{~Hz}$ and provides eye movement data for further analysis via another 166-MHz Pentium PC. Although the Eyelink II system is able to compensate for head movements, the participants did rest their heads in a chinrest to minimize head movements during experimental trials. Viewing was binocular, but eye movement data were collected only from the right eye. The participants were seated $75 \mathrm{~cm}$ from the video monitor; at this distance, one character subtended $.45^{\circ}$ of visual angle.

\section{Materials}

Two native Chinese-speaking readers first translated the Rayner and Well (1996) materials into Chinese. Those that were not easily translated were dropped, and a number of other sentences were developed. A total of 50 sentences were then rated by 24 native Chinese-speaking students at the University of Massachusetts (none of whom participated in the main experiment), by 16 Chinese students at Tianjin Normal University, and by an additional 14 native Chinese speakers from mainland China. The rating procedure provided the sentence up to where the target word should be, and the participants filled in the next word that they thought would come in the sentence. Using the resulting norming data, we ended up with 36 sentences, and each sentence had two possible target words: A given sentence could have a high- or a medium-predictable target word, a high- or a lowpredictable target word, or a medium- or a low-predictable target word. Via this procedure, there were 24 high-, 24 medium-, and 24 low-predictable target words. The mean predictability values (and the ranges) are quite comparable to those used by Rayner and Well (see Table 1). Indeed, 16 of the 36 sentences used in the present study were direct translations of the sentences used by Rayner and Well. Counterbalancing procedures ensured that each participant saw an equal number of words in the three conditions, and no sentences were repeated for a given participant.

Because we were interested in examining the effect of predictability independently of word frequency, character frequency, and character complexity, we controlled these three variables as much as possible. Of the 72 target words, 2 were 1 character, 67 were 2 characters, and 3 were 3 characters. The frequency of the first character (based on a dictionary count of $1,568,608$ characters; National Languages Committee, 1997) averaged 1,328 (range, 4-6,066; $S D=$ $1,463)$ and did not vary across predictability conditions $(p s>.25)$; likewise, the frequency of the second character averaged 1,451 (range, $2-13,167 ; S D=2,066$ ) and did not vary across predictability conditions $(p s>.27)$. Character complexity, as defined by the number of strokes per character, averaged 7.74 (range, 3-16; $S D=$ 3.2) for the first character and 7.23 (range, $2-17 ; S D=3.1$ ) for the second character and did not differ across predictability conditions $(p s>.38)$. Finally, the overall word frequency of the target words 
Table 1

Fixation Time Measures (in Milliseconds) and

\begin{tabular}{clllll}
\multicolumn{5}{c}{ Fixation Probability on the Target Word as a Function of Predictability } \\
\cline { 2 - 4 } Predictability & \multicolumn{3}{c}{ Fixation Time } \\
\cline { 2 - 4 } & FFD & Gaze & TFD & PF & PV \\
\hline High & $261(263)$ & $282(285)$ & $408(414)$ & $.75(.78)$ & $.85(.72-1.0)$ \\
R\&W & 239 & 261 & 294 & .78 & $.86(.73-1.0)$ \\
Medium & $265(269)$ & $288(292)$ & $469(471)$ & $.79(.78)$ & $.36(.11-.67)$ \\
R\&W & 240 & 261 & 301 & .88 & $.41(.13-.68)$ \\
Low & 282 & 330 & 503 & .88 & $.04(.02-.08)$ \\
R\&W & 250 & 281 & 360 & .90 & $.04(.03-.08)$ \\
\hline
\end{tabular}

Note-The values in parentheses are the data when five high-frequency words were eliminated from the analyses. The values aligned with $\mathrm{R} \& \mathrm{~W}$ represent the means from Rayner and Well (1996). For the Rayner and Well data, there was more variability in the word frequency of the target words, but they averaged 58 per million in the Francis and Kučera (1982) norms. FFD, first-fixation duration; Gaze, gaze duration; TFD, total fixation duration; PF, probability of first-pass fixation; PV, probability value (values in parentheses represent the range).

averaged 59 (range, $1-874 ; S D=146)^{4}$ and did not differ across the three levels of predictability $(p s>.09)$. The mean frequencies of the target words were comparable to those used by Rayner and Well (1996; see Table 1).

\section{Procedure}

When a participant arrived for the experiment, the eye-tracking system was calibrated. The calibration generally lasted less than $5 \mathrm{~min}$. After the calibration had been completed, the participant read the 36 sentences in a different random order, but with appropriate counterbalancing procedures to ensure that an equal number of each type of target word was read. The participant was told that the purpose of the experiment was to determine where people look as they read. He or she was also told that he or she would periodically be asked to answer comprehension questions about the sentences. These questions were asked after $25 \%$ of the 36 sentences that were read; the participants were correct over $90 \%$ of the time.

\section{RESULTS}

A number of eye movement measures were examined with respect to the target word. Specifically, the measures were (1) first-fixation duration (the duration of the first fixation on a word independent of the number of fixations on the word), (2) gaze duration (the sum of all fixations on a word prior to the reader's moving to another word), (3) total fixation time (the sum of all fixations on a word, including regressions), and (4) the probability that the reader would fixate on the target word. Table 1 shows the three fixation time measures. An ANOVA was carried out on each of the sets of data, using participants $\left(F_{1}\right)$ and items $\left(F_{2}\right)$ as random effects, and was followed up with pairwise $t$ tests.

\section{Fixation Time}

For first-fixation duration, the predictability effect was not significant by participants $\left[F_{1}(2,30)=1.26, p>\right.$ .29] but was marginally significant by items $\left[F_{2}(2,69)=\right.$ $3.064, p=.053]$. In the $t$ tests, by items, the difference between the high- and the low-predictable target words was significant $\left[t_{2}(46)=2.29, p<.05\right]$. For gaze duration, the predictability effect was significant $\left[F_{1}(2,30)=4.79\right.$, $\left.p<.05 ; F_{2}(2,69)=5.55, p<.01\right]$. In the $t$ tests, the differ- ence between high- and low-predictable targets was significant $\left[t_{1}(15)=2.35, p<.05 ; t_{2}(46)=3.03, p<.01\right]$, as was the difference between medium- and low-predictable targets $\left[t_{1}(15)=2.41, p<.05 ; t_{2}(46)=2.24, p<.05\right]$. For total time, the predictability effect was again significant $\left[F_{1}(2,30)=5.0, p<.05 ; F_{2}(2,69)=3.79, p<.05\right]$. In the $t$ tests, there were significant differences for high versus medium predictability $\left[t_{1}(15)=2.08, p=.055 ; t_{2}(46)=\right.$ $1.74, p=.089]$ and for high versus low predictability $\left[t_{1}(15)=3.07, p<.01 ; t_{2}(46)=1.94, p<.01\right]$. The comparisons for medium versus low predictability were not significant $\left[t_{1}(15)=1.1, p=.29 ; t_{2}(46)=1.02, p=.30\right]$.

\section{Fixation Probability}

The probability of a first-pass fixation on the target word indicated that the readers were more likely to skip high- and medium-predictable target words than to skip low-predictable target words. An ANOVA yielded a significant effect of predictability $\left[F_{1}(2,30)=5.4, p<.05\right.$; $\left.F_{2}(2,69)=4.6, p<.05\right]$. In the $t$ tests, there were significant differences for high versus low predictability $\left[t_{1}(15)=3.42, p<.01 ; t_{2}(46)=2.21, p<.01\right]$ and for medium versus low predictability $\left[t_{1}(15)=2.79, p<.05\right.$; $\left.t_{2}(46)=2.38, p<.05\right]$.

\section{Additional Analyses}

Although there were no significant differences across the three predictability conditions in terms of word frequency, it was the case that there were numerical differences, owing to the fact that there were some very high frequency words in the high and medium conditions. We therefore excluded five target words (three from the high and two from the medium conditions) with very high frequency counts and redid the ANOVAs. Excluding these high-frequency words resulted in mean frequencies of 27, 26, and 20 per million for the high-, medium-, and low-predictable conditions, respectively $(p \mathrm{~s}>.50)$. The means using these more restricted frequencies are shown in Table 1; obviously, the exclusion of these highfrequency items did not change the pattern of results at all. Indeed, none of the mean values for first-fixation dura- 
tion or gaze duration changed by more than $4 \mathrm{msec}$ as a result of this reanalysis. Exactly the same pattern of statistical results was present in this analysis as in the original analysis [gaze duration, $F_{1}(2,30)=3.67, p<.05$, and $F_{2}(2,64)=4.24, p<.05$; total time, $F_{1}(2,30)=5.5, p<$ .01 , and $F_{2}(2,64)=3.02, p=.056$; fixation probability, $F_{1}(2,30)=3.6, p<.05$, and $\left.F_{2}(2,64)=3.51, p<.05\right]$.

\section{Comparison With Rayner and Well (1996)}

Table 1 also shows the comparable data from Rayner and Well (1996). Whereas the fixation time measures line up reasonably well across the two studies (although the fixation times are consistently longer for the Chinese readers), so that the data patterns are similar, the fixation probability data are slightly different. That is, for the English readers, there was no difference in fixation probability between medium- and low-predictable target words; English readers were much more likely to skip over a highpredictable word than over a medium- or low-predictable word. The Chinese readers, on the other hand, were more likely to skip a high- or medium-predictable word than to skip a low-predictable word. Thus, the data pattern for the Chinese readers was the same for the fixation time measures and the fixation probability measures. Why this difference in pattern between the Chinese and the English readers emerged is not at all apparent, but it may have to do with the fact that words that are skipped are much closer to the fixation point in Chinese. Finally, the total fixation durations were markedly longer for the Chinese readers. This reflects the fact, noted earlier, that Chinese readers tend to regress more frequently than do readers of English. ${ }^{5}$

Although it is difficult to make cross-experiment comparisons, it is the case that the predictability values of the target words were very similar across the studies, the frequencies of the target words were quite similar, and the reading skill of the participants was quite similar. Although it remains quite likely that there are important cross-cultural differences, the extent to which predictability is exploited in reading seems similar across the two languages.

\section{DISCUSSION}

As we noted at the outset, data on the eye movements of Chinese readers is rather scant. It is sometimes suggested that there are differences in the way Chinese and English readers read (due to major differences in the nature of the orthographies). For example, there is some controversy regarding the relative importance of orthographic versus phonological information in the initial access of Chinese versus English words (Feng, Miller, Shu, \& Zhang, 2001; Rayner, Pollatsek, \& Binder, 1998; Wong \& Chen, 1999). Yet the more we learn about the eye movements of Chinese readers, the more apparent it becomes that there are more similarities than differences. Obviously, Chinese readers make shorter saccades than do English readers, and the perceptual span (or area of effective vision in reading) of Chinese readers is smaller than that of English readers.
But this is clearly due to the fact that informational density is much higher in Chinese than in English. Indeed, when reading rate is computed so that words per minute is the measure (rather than characters per minute), the reading rates of Chinese and English readers are quite comparable (Rayner \& Pollatsek, 1989; Sun, Morita, \& Stark, 1985).

The present study clearly shows that Chinese readers exploit contextual constraint/predictability factors in much the same way as English readers do. Other recent studies (Liu, Inhoff, Ye, \& Wu, 2002; Pollatsek, Tan, \& Rayner, 2000; Tsai et al., 2004) have demonstrated that orthographic and phonological codes are used by Chinese readers to integrate information across saccades, just as they are in English (Pollatsek, Lesch, Morris, \& Rayner, 1992). As we noted above, we observed a difference in the skipping patterns between the two groups of readers, but this may be explainable in terms of the fact that the characters are closer to fixation prior to a skip in Chinese than in English. Yang and McConkie (1999) reported that unlike readers of English, who show a clear landing position effect, wherein the eyes tend to land about halfway between the beginning and the middle of a word (the preferred viewing location; Rayner, 1979), Chinese readers do not show such an effect. Both the difference in skipping and the lack of a preferred viewing position effect may be due to the fact that the next character to be fixated is much closer to the current eye position in Chinese than in English.

In summary, although there are some clear differences between Chinese and English readers in terms of initial encoding of print (due to the nature of the logographic vs. alphabetic writing systems), once the material is encoded, reading processes appear to be more similar than dissimilar for the two groups of readers. We noted at the outset that one goal in determining how word predictability influences the eye movements of Chinese readers was to determine whether models such as E-Z Reader (Reichle et al., 1998; Reichle et al., 2003) could read Chinese. Clearly, more data need to be collected regarding character complexity, character frequency, and word frequency effects in Chinese. However, the present results (as well as the results that do exist on these other three variables) suggest that it might well be the case that the model would be effective with Chinese.

\section{REFERENCES}

Brysbaert, M., \& Vitu, F. (1998). Word skipping: Implications for theories of eye movement control in reading. In G. Underwood (Ed.), Eye guidance in reading and scene perception (pp. 125-147). Amsterdam: Elsevier, North-Holland.

Chen, H.-C., Song, H., Lau, W. Y., Wong, K. F. E., \& Tang, S. L. (2003). Developmental characteristics of eye movements in reading Chinese. In C. McBride-Chang \& H.-C. Chen (Eds.), Reading development in Chinese children (pp. 157-169). Westport, CT: Praeger.

Chen, H.-C., \& TAng, C.-K. (1998). The effective visual field in Chinese. Reading \& Writing, 10, 245-254.

Dictionary of Chinese character information (1988). Beijing: Science Press. (In Chinese)

Ehrlich, S. F., \& RAYNer, K. (1981). Contextual effects on word perception and eye movements during reading. Journal of Verbal Learning \& Verbal Behavior, 20, 641-655. 
Engbert, R., Longtin, A., \& Kliegl, R. (2002). A dynamical model of saccade generation in reading based on spatially distributed lexical processing. Vision Research, 42, 621-636.

Feng, G., Miller, K., Shu, H., \& Zhang, H. (2001). Rowed to recovery: The use of phonological and orthographic information in reading Chinese and English. Journal of Experimental Psychology: Learning, Memory, \& Cognition, 27, 1079-1101.

Francis, W. N., \& KučERA, H. (1982). Frequency analysis of English usage: Lexicon and grammar. Boston: Houghton Mifflin.

InHofF, A. W., \& LIU, W. (1997). The perceptual span during the reading of Chinese text. In H.-C. Chen (Ed.), The cognitive processing of Chinese and related Asian languages. Hong Kong: Chinese University Press.

INHOFF, A. W., \& LIU, W. (1998). The perceptual span and oculomotor activity during the reading of Chinese sentences. Journal of Experimental Psychology: Human Perception \& Performance, 24, 20-34.

KLIEGL, R., \& ENGBerT, R. (2003). SWIFT explorations. In J. Hyönä, R. Radach, \& H. Deubel (Eds.), The mind's eye: Cognitive and applied aspects of eye movement research (pp. 391-411). Oxford: Elsevier.

Kliegl, R., Grabner, E., Rolfs, M., \& Engbert, R. (2004). Length, frequency, and predictability effects of words on eye movements in reading. European Journal of Cognitive Psychology, 16, 262-284.

LiU, W., InhofF, A.W., Ye, Y., \& Wu, C. (2002). Use of parafoveally visible characters during the reading of Chinese sentences. Journal of Experimental Psychology: Human Perception \& Performance, 28, 1213-1227.

National Languages Committee (1997). Chinese Dictionary: Character and word frequency statistics report. Taipei: Author. (In Chinese)

OsAKA, N. (1993). Asymmetry of the effective visual field in vertical reading as measured with a moving window. In G. d'Y dewalle \& J. van Rensbergen (Eds.), Perception and cognition: Advances in eye movement research (pp. 275-283). Amsterdam: North-Holland.

Pollatsek, A., Bolozky, S., Well, A. D., \& Rayner, K. (1981). Asymmetries in the perceptual span for Israeli readers. Brain \& Language, 14, 174-180.

Pollatsek, A., Lesch, M., Morris, R. K., \& Rayner, K. (1992). Phonological codes are used in integrating information across saccades in word identification and reading. Journal of Experimental Psychology: Human Perception \& Performance, 18, 148-162.

PollatseK, A., TAn, L. H., \& Rayner, K. (2000). The role of phonological codes in integrating information across saccadic eye movements in Chinese character identification. Journal of Experimental Psychology: Human Perception \& Performance, 26, 607-633.

RAYNER, K. (1979). Eye guidance in reading: Fixation locations within words. Perception, 8, 21-30.

RAYNER, K. (1998). Eye movements in reading and information processing: 20 years of research. Psychological Bulletin, 124, 372-422.

Rayner, K., \& Pollatsek, A. (1989). The psychology of reading. Englewood Cliffs, NJ: Prentice-Hall.

Rayner, K., Pollatsek, A., \& Binder, K. S. (1998). Phonological codes and eye movements in reading. Journal of Experimental Psychology: Human Perception \& Performance, 24, 476-497.

RAYNER, K., \& Well, A. D. (1996). Effects of contextual constraint on eye movements in reading: A further examination. Psychonomic Bulletin \& Review, 3, 504-509.

Reichle, E. D., Pollatsek, A., Fisher, D. L., \& Rayner, K. (1998). Toward a model of eye movement control in reading. Psychological Review, 105, 125-157.

Reichle, E. D., Rayner, K., \& Pollatsek, A. (2003). The E-Z Reader model of eye-movement control in reading: Comparisons to other models. Behavioral \& Brain Sciences, 26, 445-476.

Sun, F., \& Feng, D. (1999). Eye movements in reading Chinese and English text. In J. Wang, A. W. Inhoff, \& H.-C. Chen (Eds.), Read- ing Chinese script: A cognitive analysis (pp. 189-206). Mahwah, NJ: Erlbaum.

Sun, F., Morita, M., \& Stark, L. W. (1985). Comparative patterns of reading eye movements in Chinese and English. Perception \& Psychophysics, 37, 502-506.

Tsai, J.-L., Lee, C.-Y., Tzeng, O. J. L., Hung, D. L., \& Yen, N.-S. (2004). Use of phonological codes from Chinese characters: Evidence from processing of parafoveal preview when reading sentences. Brain \& Language, 91, 235-244.

VITU, F. (1991). The influence of parafoveal processing and linguistic context on the optimal landing position effect. Perception \& Psychophysics, 50, 58-75.

Wong, K. F. E., \& CHen, H.-C. (1999). Orthographic and phonological processing in reading Chinese text: Evidence from eye fixations. Language \& Cognitive Processes, 14, 461-480.

YANG, H.-M., \& McConkIE, G. W. (1999). Reading Chinese: Some basic eye-movement characteristics. In J. Wang, A. W. Inhoff, \& H.-C. Chen (Eds.), Reading Chinese script: A cognitive analysis (pp. $207-$ 222). Mahwah, NJ: Erlbaum.

\section{NOTES}

1. There are actually two Chinese scripts: the simplified Chinese script used in mainland China and Singapore and the traditional script used in Taiwan and Hong Kong and by Chinese minorities in other countries (such as the U.S. and Canada). The experiment reported here used the simplified script, which is visually less complex and written from left to right and from top to bottom. All of our participants were from mainland China.

2. Although studies on the perceptual span in reading Chinese have not examined vertical reading, studies with Japanese readers (Osaka, 1993) have demonstrated that the perceptual span is asymmetric in the direction of reading. Likewise, Pollatsek, Bolozky, Well, and Rayner (1981) demonstrated that the perceptual span for Hebrew readers is also asymmetric to the left of fixation. Together, these studies strongly indicate that the perceptual span is primarily due to attentional factors (with the span asymmetric in the direction that the eyes will move next).

3. In other alphabetic languages, such as Dutch, French, and German, word frequency and predictability influence fixation times and skipping (Brysbaert \& Vitu, 1998; Kliegl, Grabner, Rolfs, \& Engbert, 2004; Vitu, 1991).

4. Our primary source for word frequency was the Chinese Dictionary (National Languages Committee, 1997), in which the word frequency count is based on a corpus of $1,116,417$ words. There were a few words that were not available in this frequency count, but they were available in the Dictionary of Chinese Character Information (1988), which is based on a corpus of 1,310,000 words. Because the two counts are based on slightly different numbers of words, we mathematically equated the two sources to the former frequency count.

5 . Indeed, the overall regression rate to the target word in this study averaged $16.3 \%$. Readers regressed more to the medium- $(20 \%)$ and low-predictable $(17 \%)$ target words than to the high-predictable $(12 \%)$ word $[t(15)=2.51, p<.05$, and $t(15)=1.84, p=.086$, respectively]. There was no difference between the medium and the low conditions $(t<1)$. Although the differences were only marginally significant ( $p \mathrm{~s}<$ .11 ), due to high reader variability, regressions out of the target word were more frequent for the low-predictable target word $(23 \%)$ than for the high or the medium words (17\%) in both cases. Both of these factors account for the increased total fixation durations.

(Manuscript received October 20, 2004; revision accepted for publication March 9, 2005.) 